\title{
Р.М. Гаджимирзаев
}

\section{Приближение функций, заданных на сетке $\{0, \delta, 2 \delta, \ldots\}$ суммами Фурье-Мейкснера}

Настоящая работа посвящена изучению аппроксимативных свойств частичных сумм ряда Фурье по модифицированным полиномам Мейкснера $M_{n, N}^{\alpha}(x)=M_{n}^{\alpha}(N x)(n=0,1, \ldots)$, которые при $\alpha>-1$ образуют ортогональную систему на сетке $\Omega_{\delta}=\{0, \delta, 2 \delta, \ldots\}$, где $\delta=\frac{1}{N}, N>0$ с весом $w(x)=e^{-x} \frac{\Gamma(N x+\alpha+1)}{\Gamma(N x+1)}$. Основное внимание уделено получению верхней оценки для функции Лебега указанных частичных сумм.

Библиография: 4 названия.

The present paper is devoted to the study of approximation properties of partial sums of the Fourier series in the modified Meixner polynomials $M_{n, N}^{\alpha}(x)=M_{n}^{\alpha}(N x)(n=0,1, \ldots)$ which for $\alpha>-1$ constitute an orthogonal system on the grid $\Omega_{\delta}=\{0, \delta, 2 \delta, \ldots\}$, where $\delta=\frac{1}{N}, N>0$ with weight $w(x)=e^{-x} \frac{\Gamma(N x+\alpha+1)}{\Gamma(N x+1)}$. The main attention is paid to obtaining an upper estimate for the Lebesgue function of these partial sums.

Bibliography: 4 items.

Ключевые слова: полиномы Мейкснера, ряд Фурье, функция Лебега.

Keywords: Meixner polynomials, Fourier series, Lebesgue function.

\section{Введение}

Пусть $\Omega_{\delta}=\{0, \delta, 2 \delta, \ldots\}$, где $\delta=\frac{1}{N}, N>0$. Через $M_{n, N}^{\alpha}(x)(n=0,1, \ldots)$ обозначим, следуя [1], модифицированные полиномы Мейкснера, образующие при $\alpha>-1$ ортогональную систему на дискретном множестве $\Omega_{\delta}$ c весом $w(x)=e^{-x} \frac{\Gamma(N x+\alpha+1)}{\Gamma(N x+1)}$, то есть

$$
\sum_{x \in \Omega_{\delta}} M_{n, N}^{\alpha}(x) M_{k, N}^{\alpha}(x) w(x)=\left(1-e^{-\delta}\right)^{-\alpha-1} h_{n, N}^{\alpha} \delta_{n k}, \alpha>-1,
$$

где $h_{n, N}^{\alpha}=\left(\begin{array}{c}n+\alpha \\ n\end{array}\right) e^{-n \delta} \Gamma(\alpha+1)$, а соответствующие ортонормированные с весом $\rho_{N}(x)=\left(1-e^{-\delta}\right)^{\alpha+1} w(x)$ полиномы обозначим через $m_{n, N}^{\alpha}(x)=$ $\left(h_{n, N}^{\alpha}\right)^{-\frac{1}{2}} M_{n, N}^{\alpha}(x)(n=0,1, \ldots)$. В настоящей работе для функции $f$, заданной на множестве $\Omega_{\delta}$, рассмотрена задача об исследовании аппроксимативных свойств частичных сумм $S_{n, N}^{\alpha}(f, x)$ ее ряда Фурье по полиномам $m_{n, N}^{\alpha}(x)$. Основное внимание уделено получению верхней оценки для функции Лебега $\lambda_{n, N}^{\alpha}(x)$ указанных частичных сумм при $x \in\left[0, \frac{\theta_{n}}{2}\right]$, где $\theta_{n}=4 n+2 \alpha+2$. В случае $\alpha=-\frac{1}{2}$ эта задача была решена в работе [4]. 


\section{1. Некоторые сведения о полиномах Мейкснера}

При исследовании аппроксимативных свойств частичных сумм ряда Фурье по полиномам $M_{n, N}^{\alpha}(x)$ нам понадобятся некоторые свойства этих полиномов.

Пусть $\alpha$ произвольное действительное число и $q \neq 0$. Тогда для классических полиномов Мейкснера $M_{n}^{\alpha}(x)=M_{n}^{\alpha}(x, q)$ имеют место [1]-[3]:

явное представление

$$
M_{n}^{\alpha}(x)=M_{n}^{\alpha}(x, q)=\left(\begin{array}{c}
n+\alpha \\
n
\end{array}\right) \sum_{k=0}^{n} \frac{n^{[k]} x^{[k]}}{(\alpha+1)_{k} k !}\left(1-\frac{1}{q}\right)^{k},
$$

где $x^{[k]}=x(x-1) \ldots(x-k+1),(a)_{k}=a(a+1) \ldots(a+k-1)$;

соотношение ортогональности

$$
\sum_{x=0}^{\infty} M_{n}^{\alpha}(x) M_{k}^{\alpha}(x) \rho(x)=(1-q)^{-\alpha-1} h_{n}^{\alpha}(q) \delta_{n k}, \quad 0<q<1, \alpha>-1,
$$

где $\rho(x)=\rho(x ; \alpha, q)=q^{-x} \frac{\Gamma(x+\alpha+1)}{\Gamma(x+1)}, h_{n}^{\alpha}(q)=\left(\begin{array}{c}n+\alpha \\ n\end{array}\right) q^{-n} \Gamma(\alpha+1), \delta_{n k}-$ символ Кронекера;

формула Кристоффеля-Дарбу

$$
\begin{gathered}
K_{n}^{\alpha, q}(x, y)=\sum_{k=0}^{n} q^{k} \frac{k !}{\Gamma(k+\alpha+1)} M_{k}^{\alpha}(x) M_{k}^{\alpha}(y) \\
=\frac{(n+1) ! q^{n+1}}{\Gamma(n+\alpha+1)(q-1)} \frac{M_{n+1}^{\alpha}(x) M_{n}^{\alpha}(y)-M_{n}^{\alpha}(x) M_{n+1}^{\alpha}(y)}{x-y} .
\end{gathered}
$$

Пусть $N>0, \delta=1 / N, q=e^{-\delta}$. Через $M_{n, N}^{\alpha}(x)=M_{n}^{\alpha}\left(N x, e^{-\delta}\right)(n=0,1, \ldots)$ обозначим, следуя [1], модифицированные полиномы Мейкснера, которые при $\alpha>-1$ образуют ортогональную на $\Omega_{\delta}=\{0, \delta, 2 \delta, \ldots\}$ систему с весом $\rho_{N}(x)=$ $\left(1-e^{-\delta}\right)^{\alpha+1} \rho\left(N x ; \alpha, e^{-\delta}\right)$, т.е.,

$$
\sum_{x \in \Omega_{\delta}} M_{n, N}^{\alpha}(x) M_{k, N}^{\alpha}(x) \rho_{N}(x)=h_{n, N}^{\alpha} \delta_{n k}, \quad \alpha>-1,
$$

где $h_{n, N}^{\alpha}=\left(\begin{array}{c}n+\alpha \\ n\end{array}\right) e^{n \delta} \Gamma(\alpha+1)$. Из (1.2) следует, что соответствующая ортонормированная система полиномов имеет вид $m_{n, N}^{\alpha}(x)=m_{n}^{\alpha}\left(N x, e^{-\delta}\right)=$ $\left(h_{n, N}^{\alpha}\right)^{-1 / 2} M_{n, N}^{\alpha}(x)$.

Далее, пусть

$$
K_{n, N}^{\alpha}(x, y)=\sum_{k=0}^{n} m_{k, N}^{\alpha}(x) m_{k, N}^{\alpha}(y) .
$$

Тогда в силу формулы Кристоффеля-Дарбу (1.1) мы можем записать

$$
\begin{gathered}
K_{n, N}^{\alpha}(x, y)=\frac{\delta \sqrt{(n+1)(n+\alpha+1)}}{\left(e^{\delta / 2}-e^{-\delta / 2}\right)(y-x)} \times \\
{\left[m_{n+1, N}^{\alpha}(x) m_{n, N}^{\alpha}(y)-m_{n, N}^{\alpha}(x) m_{n+1, N}^{\alpha}(y)\right]}
\end{gathered}
$$


При оценке функции Лебега нам понадобятся некоторые преобразования для $K_{n, N}^{\alpha}(x, y)$. С этой целью положим $\alpha_{n}=\sqrt{(n+1)(n+\alpha+1)}$, тогда для $n \geqslant 1$ имеем

$$
\begin{gathered}
\frac{1}{\alpha_{n}} K_{n, N}^{\alpha}(x, y)=\frac{\delta}{\left(e^{\delta / 2}-e^{-\delta / 2}\right)(y-x)} \times \\
{\left[m_{n+1, N}^{\alpha}(x) m_{n, N}^{\alpha}(y)-m_{n, N}^{\alpha}(x) m_{n+1, N}^{\alpha}(y)\right]} \\
\frac{1}{\alpha_{n-1}} K_{n, N}^{\alpha}(x, y)=\frac{\delta}{\left(e^{\delta / 2}-e^{-\delta / 2}\right)(y-x)} \times \\
{\left[m_{n, N}^{\alpha}(x) m_{n-1, N}^{\alpha}(y)-m_{n-1, N}^{\alpha}(x) m_{n, N}^{\alpha}(y)\right]+\frac{1}{\alpha_{n-1}} m_{n, N}^{\alpha}(x) m_{n, N}^{\alpha}(y)}
\end{gathered}
$$

Складывая правые и левые части равенств (1.4) и (1.5) для $n \geqslant 1$ имеем равенство

$$
\begin{gathered}
\left(\frac{1}{\alpha_{n}}+\frac{1}{\alpha_{n-1}}\right) K_{n, N}^{\alpha}(x, y)=\frac{1}{\alpha_{n-1}} m_{n, N}^{\alpha}(x) m_{n, N}^{\alpha}(y)+ \\
\frac{\delta}{\left(e^{\delta / 2}-e^{-\delta / 2}\right)(y-x)}\left[m_{n, N}^{\alpha}(y)\left(m_{n+1, N}^{\alpha}(x)-m_{n-1, N}^{\alpha}(x)\right)-\right. \\
\left.m_{n, N}^{\alpha}(x)\left(m_{n+1, N}^{\alpha}(y)-m_{n-1, N}^{\alpha}(y)\right)\right],
\end{gathered}
$$

которое мы перепишем так

$$
\begin{gathered}
K_{n, N}^{\alpha}(x, y)=\frac{\alpha_{n}}{\left(\alpha_{n}+\alpha_{n-1}\right)} m_{n, N}^{\alpha}(x) m_{n, N}^{\alpha}(y)+ \\
\frac{\alpha_{n} \alpha_{n-1}}{\left(\alpha_{n}+\alpha_{n-1}\right)} \frac{\delta}{\left(e^{\delta / 2}-e^{-\delta / 2}\right)} \frac{1}{(y-x)} \times \\
{\left[m_{n, N}^{\alpha}(y)\left(m_{n+1, N}^{\alpha}(x)-m_{n-1, N}^{\alpha}(x)\right)\right.} \\
\left.-m_{n, N}^{\alpha}(x)\left(m_{n+1, N}^{\alpha}(y)-m_{n-1, N}^{\alpha}(y)\right)\right] .
\end{gathered}
$$

Нетрудно увидеть, что равенство (1.6) справедливо также и для $n=0$.

ЗАмечание 1. Формула (1.6) впервые была получена в работе [4].

Далее, обозначим через $C\left(\Omega_{\delta}\right)$ пространство дискретных функций $f(x)$ вида $f: \Omega_{\delta} \rightarrow \mathbb{R}$ и таких, что

$$
\lim _{x \rightarrow \infty}|f(x)| e^{-x / 2}=0 .
$$

Норму в этом пространстве определим следующим образом:

$$
\|f\|_{C\left(\Omega_{\delta}\right)}=\sup _{x \in \Omega_{\delta}} e^{-x / 2}|f(x)| .
$$

Справедлива следующая лемма 
ЛЕмма 1.1. Пусть $\alpha>-1, p>1, l_{\rho_{N}}^{p}-$ пространство функиий, заданных на $\Omega_{\delta}$ и такux, что

$$
\|f\|_{l_{\rho_{N}}^{p}}=\left(\sum_{x \in \Omega_{\delta}}|f(x)|^{p} \rho_{N}(x)\right)^{1 / p}<\infty .
$$

Тогда $C\left(\Omega_{\delta}\right) \subset l_{\rho_{N}}^{p}$ nрu $1<p<2$.

Из леммы 1.1 следует, что для произвольной функции $f \in C\left(\Omega_{\delta}\right)$ мы можем определить коэффициенты Фурье-Мейкснера

$$
f_{k}^{\alpha}=\sum_{t \in \Omega_{\delta}} f(t) m_{k, N}^{\alpha}(t) \rho_{N}(t)
$$

и ряд Фурье-Мейкснера

$$
f(x) \sim \sum_{k=0}^{\infty} f_{k}^{\alpha} m_{k, N}^{\alpha}(x) .
$$

Через $S_{n, N}^{\alpha}(f, x)$ обозначим частичную сумму ряда (1.7):

$$
S_{n, N}^{\alpha}(f, x)=\sum_{k=0}^{n} f_{k}^{\alpha} m_{k, N}^{\alpha}(x)
$$

которую в силу (1.3) можем представить в виде

$$
S_{n, N}^{\alpha}(f, x)=\sum_{t \in \Omega_{\delta}} f(t) \mathcal{K}_{n, N}^{\alpha}(t, x) e^{-t} \frac{\Gamma(N t+\alpha+1)}{\Gamma(N t+1)}\left(1-e^{-\delta}\right)^{\alpha+1} .
$$

Будем рассматривать $S_{n, N}^{\alpha}(f, x)$ как аппарат приближения функций из $C\left(\Omega_{\delta}\right)$. Для $f \in C\left(\Omega_{\delta}\right)$ через $E_{n}(f)$ обозначим наилучшее приближение $f$ в метрике пространства $C\left(\Omega_{\delta}\right)$ алгебраическими полиномами степени $n$, то есть,

$$
E_{n}(f)=\inf _{p_{n} \in H^{n}}\left\|f-p_{n}\right\|_{C\left(\Omega_{\delta}\right)}
$$

где $H^{n}$ - подпространство алгебраических полиномов $p_{n}(x)$ степени не выше $n$. Пусть, далее $p_{n}(f)=p_{n}(f, x)$ полином наилучшего приближения к функции $f$ в $C\left(\Omega_{\delta}\right)$, для которого $E_{n}(f)=\left\|f-p_{n}(f)\right\|_{C\left(\Omega_{\delta}\right)}$. Тогда, пользуясь тем, что для $p_{n} \in H^{n}$ будет $S_{n, N}^{\alpha}\left(p_{n}\right)=p_{n}$ мы можем записать

$$
\begin{gathered}
\left|f(x)-S_{n, N}^{\alpha}(f, x)\right|=\left|f(x)-p_{n}(f, x)+p_{n}(f, x)-S_{n, N}^{\alpha}(f, x)\right| \leqslant \\
\left|f(x)-p_{n}(f, x)\right|+\left|S_{n, N}^{\alpha}\left(p_{n}-f, x\right)\right| .
\end{gathered}
$$

Отсюда и из (1.8)

$$
\begin{gathered}
e^{-\frac{x}{2}}\left|f(x)-S_{n, N}^{\alpha}(f, x)\right| \leqslant e^{-\frac{x}{2}}\left|f(x)-p_{n}(f, x)\right|+e^{-\frac{x}{2}}\left|S_{n, N}^{\alpha}\left(p_{n}-f, x\right)\right| \leqslant \\
E_{n}(f)\left(1+\lambda_{n, N}^{\alpha}(x)\right),
\end{gathered}
$$


где

$$
\lambda_{n, N}^{\alpha}(x)=\sum_{t \in \Omega_{\delta}} e^{-\frac{t+x}{2}} \frac{\Gamma(N t+\alpha+1)}{\Gamma(N t+1)}\left(1-e^{-\delta}\right)^{\alpha+1}\left|\mathcal{K}_{n, N}^{\alpha}(t, x)\right| .
$$

В связи с неравенством (1.9) возникает задача об оценке на $[0, \infty)$ функции Лебега $\lambda_{n, N}^{\alpha}(x)$, определяемой равенством (1.10). Чтобы избежать чрезмерного объема статьи, мы ограничимся рассмотрением этой задачи для $x \in G_{1}=\left[0, \frac{3}{\theta_{n}}\right]$ и $x \in G_{2}=\left[\frac{3}{\theta_{n}}, \frac{\theta_{n}}{2}\right]$, оставляя случай $x \in\left(\frac{\theta_{n}}{2}, \infty\right)$ в качестве объекта исследования другой работы. Основным результатом настоящей работы является следующая теорема.

Tеорема 1.1. Пусть $\alpha>-1, \theta_{n}=4 n+2 \alpha+2, \lambda>0,1 \leqslant n \leqslant \lambda N$. Тогда имеют место следующие оценки:

1) если $x \in G_{1}=\left[0, \frac{3}{\theta_{n}}\right]$, mo

$$
\lambda_{n, N}^{\alpha}(x) \leqslant c(\alpha, \lambda)\left\{\begin{array}{ll}
1, & -1<\alpha<-\frac{1}{2}, \\
\ln (n+1), & \alpha=-\frac{1}{2}, \\
n^{\alpha+\frac{1}{2}}, & \alpha>-\frac{1}{2} .
\end{array} ;\right.
$$

2) если $x \in G_{2}=\left[\frac{3}{\theta_{n}}, \frac{\theta_{n}}{2}\right]$, mo

$$
\lambda_{n, N}^{\alpha}(x) \leqslant c(\alpha, \lambda) \begin{cases}\ln (n x+1), & -1<\alpha<-\frac{1}{2}, \\ \ln (n+1), & \alpha=-\frac{1}{2}, \\ \ln (n+1)+\left(\frac{n}{x}\right)^{\frac{\alpha}{2}+\frac{1}{4}}, & \alpha>-\frac{1}{2} .\end{cases}
$$

\section{Список литературы}

[1] Шарапудинов И.И. Многочлены, ортогональные на сетках. Махачкала: Изд-во Даг. гос. пед. ун-та. 1997.

[2] Бейтмен Г., Эрдейи А. Высшие трансцендентные функции. Т. 2. М.: Наука. 1974.

[3] Никифоров А.Ф., Суслов С.К., Уваров В.Б. Классические ортогональные многочлены дискретной переменной. М.: Наука. 1985.

[4] Гаджиева 3. Д. Смешанные ряды по полиномам Мейкснера. Кандидатская диссертация - Саратов. Саратовский гос. ун-т. 2004. 